\title{
Skin Lesion Segmentation using Deep Hypercolumn Descriptors
}

Dhanesh Ramachandram

Graham W. Taylor
School of Engineering, University of Guelph

School of Engineering, University of Guelph and Vector Institute

\section{Abstract}

We present a image segmentation method based on deep hypercolumn descriptors which produces state-of-the-art results for the segmentation of several classes of benign and malignant skin lesions. We achieve a Jaccard index of 0.792 on the 2017 ISIC Skin Lesion Segmentation Challenge dataset.

\section{Introduction}

One of the fundamental and most challenging tasks in digital image analysis is semantic segmentation, which is the process of assigning pixel-wise labels to regions in an image that share some high-level semantics. In this paper, we focus on the task of accurately segmenting benign and malignant skin lesions in dermatoscopy images as a means of lesion quantification. Among the skin lesions considered in our work is melanoma which is an aggressive malignant tumour originating from melanocytes cells - skin cells responsible for the production of melanin. The American Cancer Society estimates that in 2017, in the United States alone, more than 87,000 new melanoma cases will be diagnosed with an estimated 9,300 fatalities [1]. Skin melanoma lesions share similar visual characteristics with other benign skin lesions such as nevus and seborrhoeic keratosis as shown in Fig. 1 .

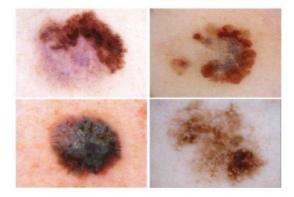

Melanoma

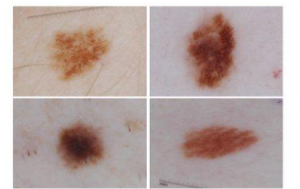

Nevus

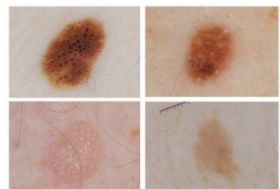

Sebborhoeic Keratosis
Fig. 1: Examples of various skin benign and malignant skin lesions

Skin lesion segmentation is challenging due to a variety of factors, such as variations in skin tone, uneven illumination, partial occlusion due to the presence of hair, low contrast between lesion and surrounding skin, and the presence of freckles or gauze in the image frame, which may be mistaken for lesions. A successful lesion segmentation technique should be robust enough to accommodate this variation. Fig. 2 shows an example dermatoscope image of a skin lesion and its corresponding binary mask.
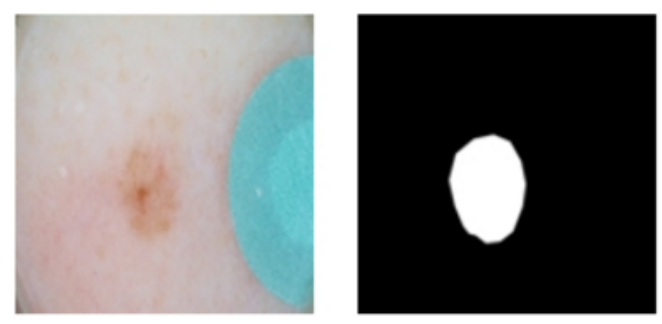

Fig. 2: Left: An example of a skin lesion image with a blue fiducial marker in the background. Right: The corresponding ground truth binary segmentation mask.

\section{Related Work}

Skin lesion segmentation is a widely researched topic in medical image analysis[2]. Until recently, most skin lesion segmentation approaches were based on meticulously designed image features 3 4 5]. Such approaches often require extensive pre-processing and post-processing approaches such as hair removal, edge-preserving smoothing and morphological operations. Therefore the robustness of such approaches could be limited as each new scenario may require custom tuning.

An alternative approach to manually crafting image features for segmentation is to instead leverage deep neural networks to automatically learn robust image features given sufficient labeled examples. Deep learning is becoming the dominant approach for many medical imaging problems $[6]$ and has seen tremendous success for the related skin lesion classification task[7]. Early deep learning approaches to image segmentation used a patch-wise training strategy [8, 9], where overlapping patches are used to train a convolutional neural network to predict the value of the pixel centered on each patch. While this approach overcame the requirement of having a large labeled dataset, the approach was computationally inefficient due to obvious redundancies in information contained in overlapping patches. Long et al. [10] proposed the Fully Convolutional Neural Network (FCN) architecture which has become the mainstream approach to deep semantic segmentation and many variants [11, 12, 13] have been proposed since. In FCNs, the usual fully-connected and final prediction layers of convolutional neural networks (CNNs) are replaced with convolutional layers to facilitate dense prediction. In order to learn contextual information contained in images, CNNs use pooling operations (e.g. max-pooling) or strided-convolutions, that produce downsampled outputs across the layers of the network resulting in a much smaller prediction mask. Therefore, FCNN architectures require a single upsampling or several progressive upsampling or "de-convolution" layers to upscale the pixel-wise predictions of the network to match the dimensions of the input image. In the 2017 skin lesion segmentation challenge (ISIC2017: Skin Lesion Analysis Towards Melanoma Detection), $70 \%$ of the submissions, and 9 out of the top 10 submissions employed deep learning strategies for segmentation.

Deep learning architectures often require a large labelled dataset, which is uncommon in the medical domain. Recently, transfer learning approaches (i.e. fine-tuning a pre-trained network on a limited, but different dataset) has been successful[14, 15, 16]. Nevertheless, the mechanisms of transfer learning and why such approaches work on vastly different domains has been challenging to interpret[17].

Our approach to skin lesion segmentation is based on the idea of using hypercolumn descriptors first proposed by[18]. Hypercolumn descriptors for a given pixel are formed by extracting activations from multiple convolutional layers of a CNN that correspond to the same pixel. These multi-scale descriptors, which capture rich semantics and localization information, can then be used to train a non-linear classifier to perform pixel-wise predictions. Hypercolumn descriptors have been applied to problems such as semantic segmentation[18], edge detection, surface normal estimation[19] and auto-colourization of grayscale images[20]. We demonstrate its effectiveness for the challenging skin lesion segmentation problem and show state-of-the-art performance on the ISIC2017 Skin Lesion Segmentation Challenge ${ }^{1}$ which is larger and more challenging than the previous dataset used in a similar challenge (ISBI 2016). The training dataset consists of 2000 dermatoscopy images of three types of skin lesions: nevus, seborrhoeic keratosis and melanoma - the latter lesion being malignant — and their binary masks. The masks were created by an expert clinician, using either a semiautomated process (using a user-provided seed point, a user-tuned flood-fill algorithm, and morphological filtering) or a manual process (from a series of user-provided polyline points). Fig. 2 shows an example lesion and its corresponding binary mask.

\section{Methodology}

Our skin lesion segmentation model is an adaptation of the PixelNet architecture[19]. The PixelNet architecture uses the convolutional layers of the VGG16 [21] architecture to form hypercolumn descriptors using sparsely sampled pixels from input images during training.

1 http://challenge2017.isic-archive.com 
These descriptors are then used to train a 2-layered multi-layer perceptron (MLP) to perform pixel-wise prediction. We demonstrate that it is possible to achieve state-of-the-art segmentation performance by training the network from scratch using a relatively small dataset.

\subsection{Preprocessing}

For this application, the input images and the corresponding groundtruth masks are first resized to 224 by 224 pixels to match the resolution of images in the pre-training stage. When using a pretrained VGG16 net to extract the hypercolumns, we retained the normalization of the input images using the mean channel-wise pixel intensities computed for the entire ImageNet dataset. We perform data augmentation on-the-fly by randomly rotating both the image and its mask by 90 -degree increments as well as flipping the images. In addition, we also randomly varied the image brightness, hue and contrast (within a small range) for each minibatch.

\subsection{Deep Hypercolumns}

During the training phase, we feed the input image to a VGG16 network and extract the sparse hypercolumn descriptors from selected convolutional layers. The hypercolumns are formed by concatenating a series of activations of the convolutional layers. In our implementation, we chose the activations from the final convolutional layer from each convolutional and fully-connected block in the VGG16 architecture (i.e. $\operatorname{conv} 1_{2}, \operatorname{conv} 2_{2}, \operatorname{conv} 3_{3}, \operatorname{conv} 4_{3}, \operatorname{conv} 5_{3}$, and $F C_{2}$ layers.) to form the hypercolumn. The fully connected layers in the original VGG16 network are implemented as $1 \times 1$ convolution layers. As each convolutional block is preceded by a max-pooling operation that downsamples the activations, we perform bilinear upsampling using an appropriate scaling factor such that the resulting resolution for the activations of each layer forming the hypercolumn is $224 \times 224$. Then, we sparsely sample random points from the dense hypercolumns to form rich descriptors for a given pixel in the input image. The sparse hypercolumn descriptors are then fed to a non-linear classifier, in our case, a 2-layered MLP (again, implemented as $1 \times 1$ convolutions) with 4096 and 2048 neurons respectively. We use a sparsely-sampled output mask, whose pixels correspond to the location of the sparse hypercolumns to learn pixel-wise class predictions.

\subsection{Training}

We implemented our network using TensorFlow [22 and experimented both fine-tuning an ImageNet-pretrained VGG16 net as well as training the entire network from scratch. In both cases, we used batch normalization and ADAM optimization with an initial learning rate of $10^{-3}$ using a per-pixel cross-entropy loss function. Since we construct hypercolumn descriptors from sparsely sampled pixels, we empirically found that using a sample size of 1600 pixels from a batch size of 5 images provided best results. Training typically converges after 120 epochs on a NVIDIA Titan-Xp GPU with $12 \mathrm{~Gb}$ of RAM. This takes around 3 hours. During inference, we turn off sparse random sampling and use the dense hypercolumns for image segmentation.

\section{Results and Discussion}

Example segmentation outputs from our lesion segmentation architecture are shown in Fig. 4. It can be seen that the model produces accurate segmentations for a wide range of skin lesion appearances in the dermatoscopy images. Tab. 1 shows the comparative results of our approach against the top-3 scoring participants of the 2017 ISIC lesion segmentation challenge. Our model achieves significantly higher Jaccard score than the best submissions for the 2017 ISIC skin lesion segmentation challenge and can be effectively trained from scratch using a relatively small dataset. The ranking of segmentation quality is based on the Jaccard index, as used in the challenge, which measures the degree of overlap between the predicted segmentation and the expert-annotated ground truth masks. It is defined as: $J A=\frac{T P}{T P+F N+F P}$ where $T P$ is the number of True Positives, $F N$ is the number of False Negatives and $F P$ is the number of False Positives. We found that a network trained from scratch produces significantly better segmentation performance as opposed to fine-tuning a pre-trained network. This may be attributed to the misalignment between the distribution of images in ImageNet and the distribution of dermatoscopy images in our target dataset.

Despite the impressive results obtained for the skin lesion segmentation, the model has a large number of parameters and the computation of dense hypercolumns during inference is computeintensive. We are currently working to reduce the model size and inference times so that a relatively lean and fast model can be deployed on mobile-phones.

\section{Acknowledgements}

We thank OCE and NSERC for jointly funding this research project, and CFI for funding computational infrastructure. We also thank NVIDIA for the generous hardware donation for use in the project.

\section{References}

[1] American Cancer Society. Key statistics for melanoma skin cancer, 2017. Accessed: 25 Feb 2017.

[2] M Emre Celebi, Quan Wen, Hitoshi lyatomi, Kouhei Shimizu, Huiyu Zhou, and Gerald Schaefer. A state-of-the-art survey on lesion border detection in dermoscopy images, 2015.

[3] Huiyu Zhou, Gerald Schaefer, M Emre Celebi, Faquan Lin, and Tangwei Liu. Gradient vector flow with mean shift for skin lesion segmentation. Computerized Medical Imaging and Graphics, 35(2):121-127, 2011

[4] Xiaojing Yuan, Ning Situ, and George Zouridakis. A narrow band graph partitioning method for skin lesion segmentation. Pattern Recognition, 42(6):1017-1028, 2009.

[5] Gerald Schaefer, Maher I Rajab, M Emre Celebi, and Hitoshi lyatomi. Colour and contrast enhancement for improved skin lesion segmentation. Computerized Medical Imaging and Graphics, 35(2):99-104, 2011.

[6] Ge Wang. A perspective on deep imaging. IEEE Access, 4:8914-8924, 2016

[7] Andre Esteva, Brett Kuprel, Roberto A Novoa, Justin Ko, Susan M Swetter, Helen M Blau, and Sebastian Thrun. Dermatologist-level classification of skin cancer with deep neural networks. Nature, 542(7639):115-118, 2017

[8] Feng Ning, Damien Delhomme, Yann LeCun, Fabio Piano, Léon Bottou, and Paolo Emilio Barbano. Toward automatic phenotyping of developing embryos from videos. IEEE Transactions on Image Processing, 14(9):1360-1371, 2005.

[9] Dan Ciresan, Alessandro Giusti, Luca M Gambardella, and Jürgen Schmidhuber. Deep neural networks segment neuronal membranes in electron microscopy images. In Advances in neural information processing systems, pages 2843-2851, 2012.

[10] Jonathan Long, Evan Shelhamer, and Trevor Darrell. Fully convolutional networks for semantic segmentation. In Proceedings of the IEEE Conference on Computer Vision and Pattern Recognition, pages 3431-3440, 2015.

[11] Vijay Badrinarayanan, Alex Kendall, and Roberto Cipolla. Seg net: A deep convolutional encoder-decoder architecture for image segmentation. arXiv preprint arXiv:1511.00561, 2015.

[12] Liang-Chieh Chen, George Papandreou, lasonas Kokkinos, Kevin Murphy, and Alan L Yuille. Deeplab: Semantic image segmentation with deep convolutional nets, atrous convolution, and fully connected crfs. arXiv preprint arXiv:1606.00915, 2016.

[13] Olaf Ronneberger, Philipp Fischer, and Thomas Brox. U-net: Convolutional networks for biomedical image segmentation. In International Conference on Medical Image Computing and Computer-Assisted Intervention, pages 234-241. Springer, 2015. 


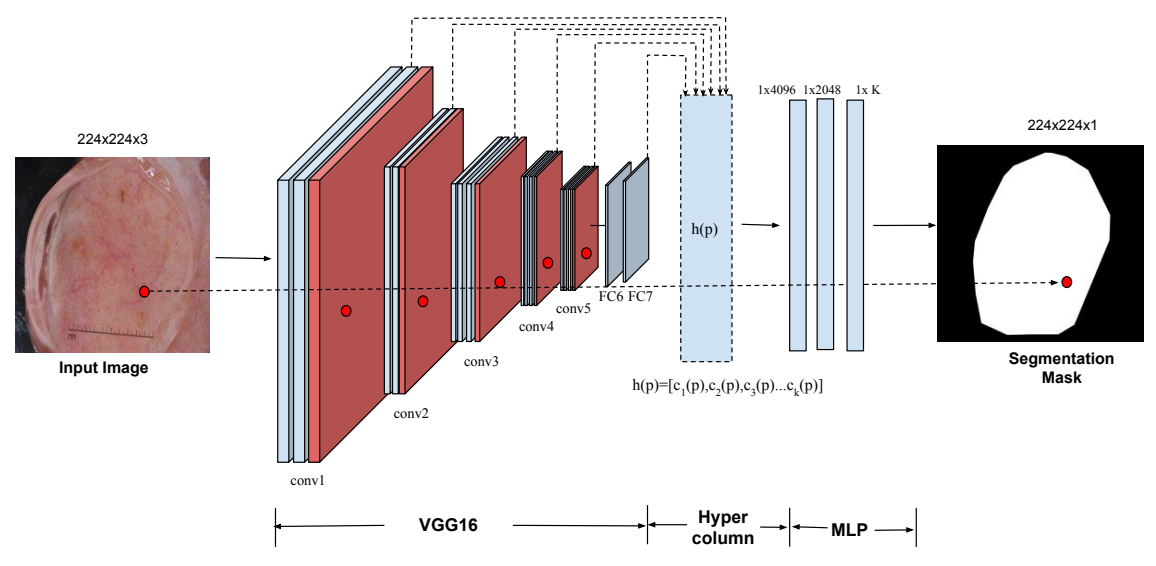

Fig. 3: An illustration of the network architecture used in this work. The hypercolumn descriptors are constructed by concatenating a series of multi-scale activations from convolutional layers of a VGG16 net.

Table 1: Results for 2017 ISIC Skin Segmentation Challenge dataset against the top-3 submissions

\begin{tabular}{lllllll}
\hline Ref. & Method & Fine-tuned & Accuracy & Jaccard Index & Sensitivity & Specificity \\
\hline Ours & Deep hypercolumn descriptors & No & $\mathbf{0 . 9 1 6}$ & $\mathbf{0 . 7 9 2}$ & $\mathbf{0 . 7 9 0}$ & $\mathbf{0 . 9 5 4}$ \\
Ours & Deep hypercolumn descriptors & Yes & 0.900 & 0.769 & 0.783 & 0.937 \\
{$[23]$} & Fully convolutional-deconvolutional & No & 0.934 & 0.765 & 0.825 & 0.975 \\
{$[24]$} & U-Net & No & 0.932 & 0.762 & 0.820 & 0.978 \\
{$[25]$} & ResNet (with additional 8K training images) & Yes & 0.934 & 0.760 & 0.802 & 0.985 \\
\hline
\end{tabular}
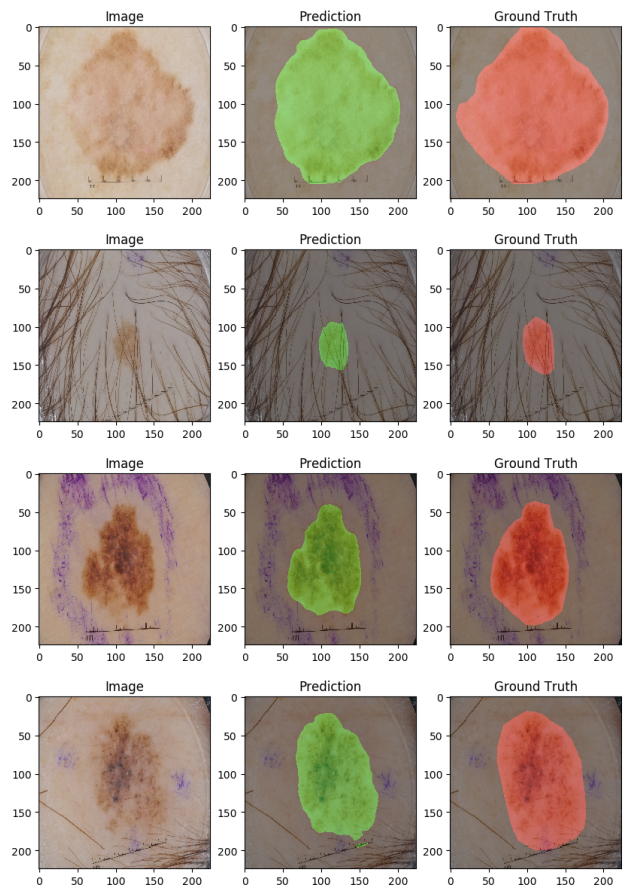

Fig. 4: Examples of segmentation output using our approach

[14] Annegreet Van Opbroek, M Arfan Ikram, Meike W Vernooij, and Marleen De Bruijne. Transfer learning improves supervised image segmentation across imaging protocols. IEEE transactions on medical imaging, 34(5):1018-1030, 2015.

[15] Afonso Menegola, Michel Fornaciali, Ramon Pires, Flávia Vasques Bittencourt, Sandra Avila, and Eduardo Valle. Knowledge transfer for melanoma screening with deep learning. arXiv preprint arXiv:1703.07479, 2017.

[16] Jeremy Kawahara, Aicha BenTaieb, and Ghassan Hamarneh. Deep features to classify skin lesions. In Biomedical Imaging
(ISBI), 2016 IEEE 13th International Symposium on, pages 1397-1400. IEEE, 2016.

[17] Hariharan Ravishankar, Prasad Sudhakar, Rahul Venkataramani, Sheshadri Thiruvenkadam, Pavan Annangi, Narayanan Babu, and Vivek Vaidya. Understanding the mechanisms of deep transfer learning for medical images. arXiv preprint arXiv:1704.06040, 2017.

[18] Bharath Hariharan, Pablo Arbeláez, Ross Girshick, and Jitendra Malik. Hypercolumns for object segmentation and finegrained localization. In Proceedings of the IEEE Conference on Computer Vision and Pattern Recognition, pages 447-456, 2015.

[19] Aayush Bansal, Xinlei Chen, Bryan Russell, Abhinav Gupta, and Deva Ramanan. Pixelnet: Towards a general pixel-level architecture. arXiv preprint arXiv:1609.06694, 2016.

[20] Gustav Larsson, Michael Maire, and Gregory Shakhnarovich. Learning representations for automatic colorization. In European Conference on Computer Vision, pages 577-593. Springer, 2016.

[21] Karen Simonyan and Andrew Zisserman. Very deep convolutional networks for large-scale image recognition. arXiv preprint arXiv:1409.1556, 2014.

[22] Martín Abadi, Ashish Agarwal, Paul Barham, Eugene Brevdo, Zhifeng Chen, Craig Citro, Greg S Corrado, Andy Davis, Jeffrey Dean, Matthieu Devin, et al. Tensorflow: Large-scale machine learning on heterogeneous distributed systems. arXiv preprint arXiv:1603.04467, 2016.

[23] Yading Yuan, Ming Chao, and Yeh-Chi Lo. Automatic skin lesion segmentation with fully convolutional-deconvolutional networks. arXiv preprint arXiv:1703.05165, 2017.

[24] Matt Berseth. Isic 2017-skin lesion analysis towards melanoma detection. arXiv preprint arXiv:1703.00523, 2017.

[25] Lei Bi, Jinman Kim, Euijoon Ahn, and Dagan Feng. Automatic skin lesion analysis using large-scale dermoscopy images and deep residual networks. arXiv preprint arXiv:1703.04197, 2017. 